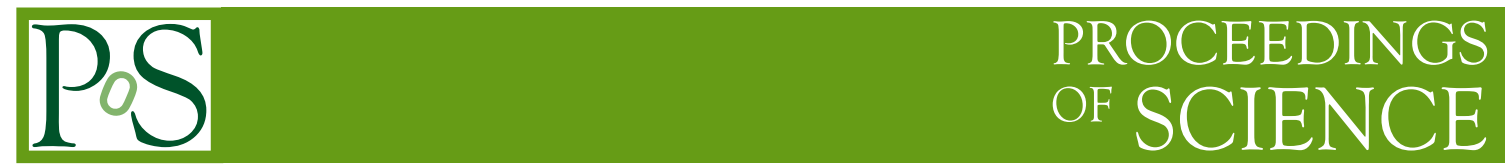

\title{
High Energy Jets at the LHC
}

\section{Jack Medley*}

Higgs Centre, University of Edinburgh, Mayfield Road, Edinburgh, EH9 $3 J G$

E-mail: jack.medley@ed.ac.uk

The High Energy Jets (HEJ) framework systematically resums the large logarithms which enhance the high order terms in the perturbation theory of QCD which arise in the study of wideangle jet production the LHC. Our approximation is valid in the limit of hard outgoing partons all well separated in rapidity and is easily generalised to a wide range of LHC phenomena, including observables with multiple high energy jets accompanied by an electroweak boson. Some recent results comparing HEJ to experimental data is presented to highlight the importance of these enhanced terms.

XXII. International Workshop on Deep-Inelastic Scattering and Related Subjects, 28 April - 2 May 2014

Warsaw, Poland

${ }^{*}$ Speaker. 


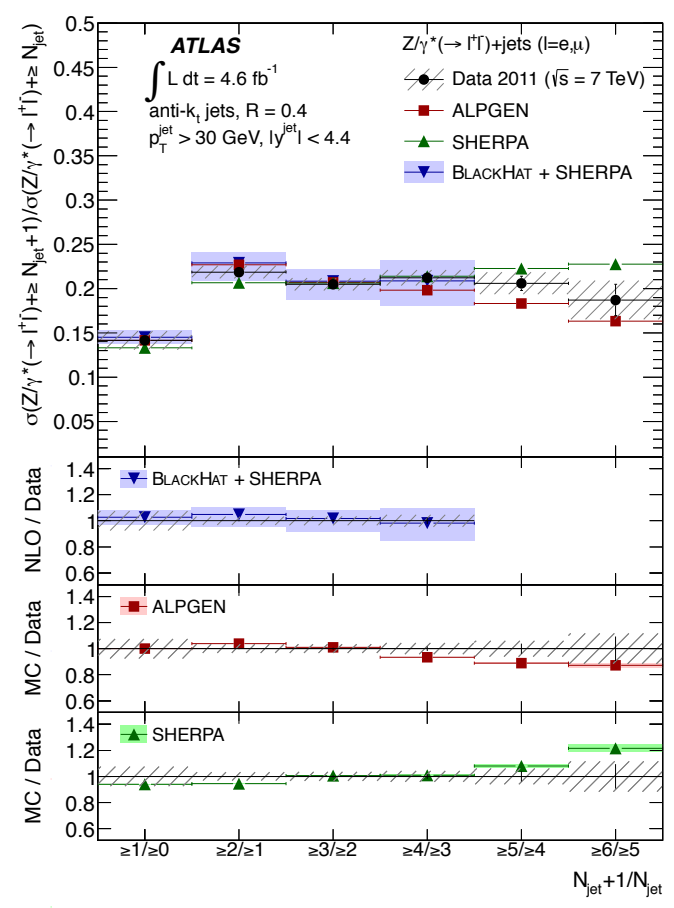

Figure 1: The $\frac{n+1}{n}$-jet rate in the $Z+$ jets channel as measured by the ATLAS experiment.

\section{Introduction}

Understanding jet physics is essential both for improving precision measurements of Standard Model physics and for searching for new physics at present and future hadronic colliders such as the LHC. Of particular interest are events in which jets appear in conjunction with electroweak bosons. For example, the production of $Z^{0}+$ jets at the LHC give us an opportunity for a precision test of QCD as well as being a background to other for the measurements of the Higgs boson. A very high jet multiplicity has been observed in the $Z^{0}+$ jets by the ATLAS collaboration (see figure [1]) [5] and so it is also crucial that we have a good understanding of high multiplicity QCD amplitudes.

\section{High Energy Jets}

In the limit of large invariant mass, $s_{i j}=2 p_{i} \cdot p_{j}$, between outgoing partons in a QCD scattering amplitude (the 'High Energy Limit') the traditional approach of perturbatively expanding observables in the strong coupling constant $\alpha_{s}$ begins to break down since each term is accompanied by a large logarithm, $\ln \left(\frac{s}{-t}\right)$, where $s$ and $t$ are the usual Mandelstam variables. The high energy limit can also be stated in terms of the rapidities, $y_{i}$, and perpendicular momentum components, $p_{i \perp}$, of the outgoing partons (the 'Multi-Regge Kinematic Limit'):

$$
y_{1} \gg y_{2} \gg \cdots \gg y_{n}, \quad\left|p_{i \perp}\right| \sim\left|p_{j \perp}\right| \quad \forall i, j
$$




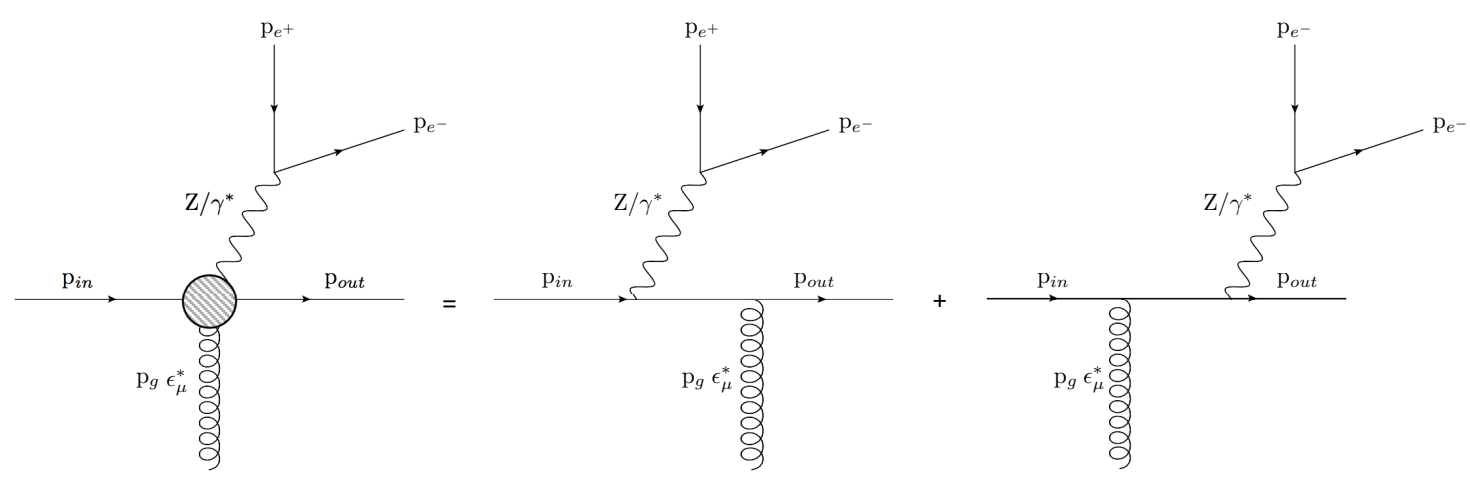

Figure 2: The diagrammatic form for the 'current' describing emission of a $Z / \gamma^{*}$.

and in terms of this limit it is clear that the region where large logarithms dominate is that of wideangle hard scattering. In this limit it is possible [1][2] to write down a factorised form for $2 \rightarrow n$ matrix element as follows

$$
\begin{array}{r}
\left|\mathscr{A}_{a b \rightarrow a g g \cdots g b}\right|^{2}=\frac{1}{4\left(N_{c}^{2}-1\right)}|| S_{a b \rightarrow a b} \|^{2}\left(\frac{g_{s}^{2} C_{X}}{t_{i}}\right)\left(\frac{g_{s}^{2} C_{Y}}{t_{n-1}}\right) \\
\prod_{i=1}^{n-2}\left(-\frac{g_{s}^{2} C_{A}}{t_{i} t_{i+1}} V_{\mu}\left(q_{i}, q_{i+1}\right) V^{\mu}\left(q_{i}, q_{i+1}\right)\right) \prod_{j=1}^{n-2} \exp \left[\omega^{0}\left(q_{j}\right)\left(y_{j-1}-y_{j}\right)\right]
\end{array}
$$

where $q_{1}=p_{a}-p_{1}, q_{i+1}=q_{i}-p_{i}$ and $t_{i}=q_{i}^{2}$. The 'spinor string', $\left\|S_{a b \rightarrow a b}\right\|^{2}$, is given by:

$$
\left\|S_{a b \rightarrow a b}\right\|^{2}=\sum_{\text {polarisation }}\left|j_{1}\left(p_{a}, p_{1}\right) \cdot j_{2}\left(p_{b}, p_{n}\right)\right|^{2},
$$

and comprises exact $2 \rightarrow 2$ scattering amplitude for the hard incoming partons $a$ and $b$ (summed and averaged over incoming and outgoing helicities/polarisations). $V_{\mu}\left(q_{i}, q_{i+1}\right)$ is the aforementioned effective vertex for the inclusion of extra gluon emissions in the final state and the product of exponentials encodes the large logarithms described above. The above expression is readily generalised to more complicated final states involving electroweak bosons simply by the modification of the spinor string. For example, in the case where a $Z^{0}$ is emitted from the forward-incoming quark, $p_{a / 1}, j_{1}\left(p_{a}, p_{1}\right)$ would be modified to a slightly more complicated form, shown diagrammatically in figure (2), given by

$$
j_{\mu}^{Z}=\bar{u}^{h_{1}}\left(p_{1}\right)\left(\gamma^{\sigma} \frac{\not p_{1}+\not p_{Z}}{\left(p_{1}+p_{Z}\right)^{2}} \gamma_{\mu}+\gamma_{\mu} \frac{\not p_{a}-\not p_{Z}}{\left(p_{a}-p_{Z}\right)^{2}} \gamma_{\sigma}\right) u^{h_{a}}\left(p_{a}\right) \times \bar{u}^{h_{e^{-}}}\left(p_{e^{-}}\right) \gamma_{\sigma} u^{h_{e^{+}}}\left(p_{e^{+}}\right)
$$

As indicated in figure (2) we also include the contribution from an off-shell photon being exchanged in place of the $Z^{0}$ and the interference between the two channels. The HEJ package also includes all of the contributions not included in our resummation up to and including states with 4 final state jets by matching the leading order matrix element program MadGraph [7]. 

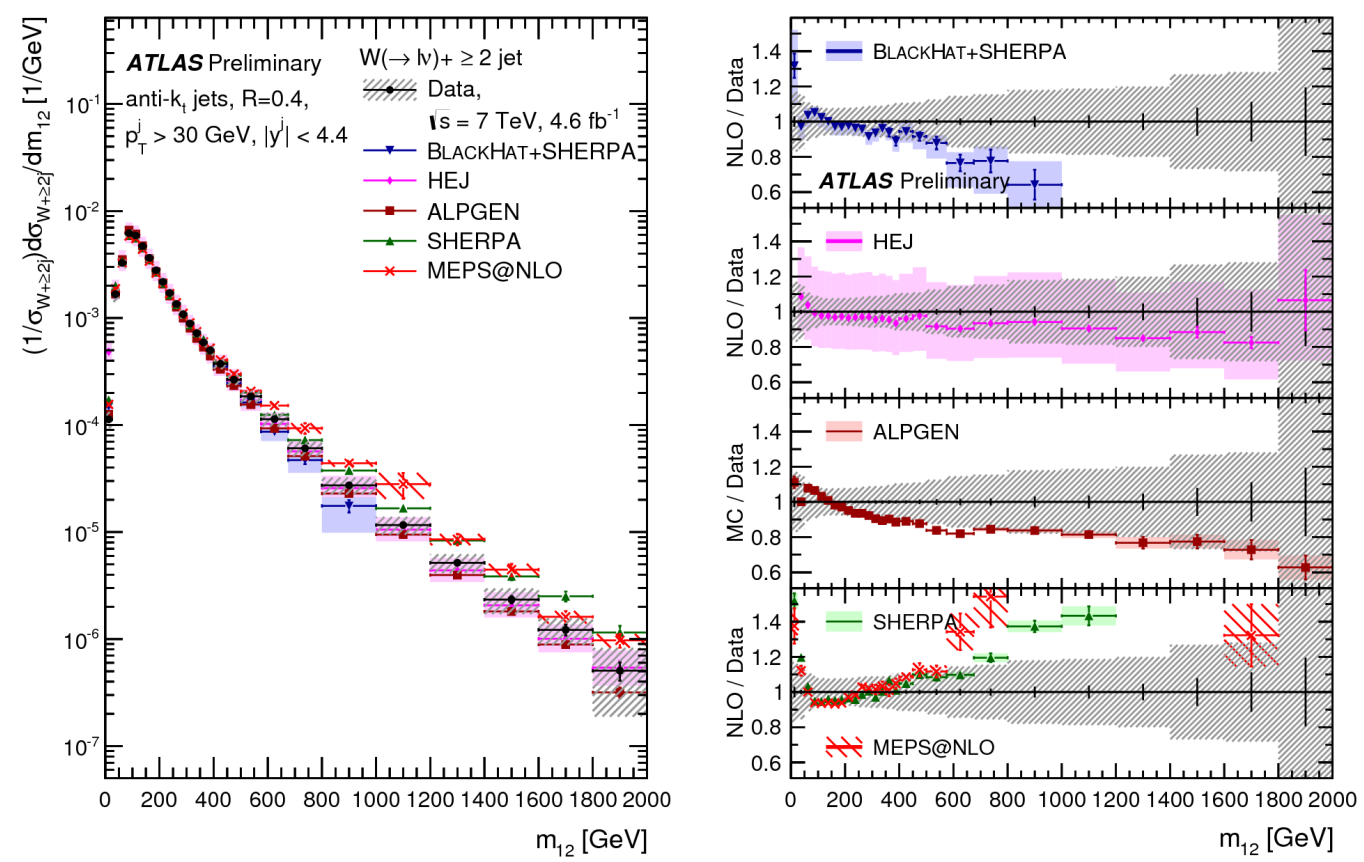

Figure 3: $\mathrm{W}+\mathrm{jets}$ cross section as a function of the dijet invariant mass between the two leading jets.

\section{Comparisons}

There have been many very detailed analyses done in recent years comparing HEJ to data (or to other Monte Carlos). Here we present a recent result showing the importance of the enhanced high order corrections included in our resummation. A recent $W^{ \pm}+$jets analysis by the ATLAS collaboration [6], see figure (3), shows that in the limit of large invariant mass discussed above HEJ describes data well where other fixed-order tools struggle. It is also important to note that even at small values for $m_{12}$ (i.e. far outside the high energy limit) HEJ describes the data very well.

\section{References}

[1] J. R. Andersen and J. M. Smillie, JHEP 1001 (2010) 039.

[2] J. R. Andersen and J. M. Smillie, JHEP 1106 (2011) 010.

[3] J. R. Andersen, T. Hapola and J. M. Smillie, JHEP 1209 (2012) 047.

[3] J. R. Andersen and J. M. Smillie, Phys. Rev. D 81, 114021 (2010).

[4] J. R. Andersen, L. Lonnblad and J. M. Smillie, JHEP 1107 (2011) 110.

[5] G. Aad et al. [ATLAS Collaboration], JHEP 1307 (2013) 032

[6] G. Aad et al. [ATLAS Collaboration], ATLAS-CONF-2014-035 (2014)

[7] J. Alwall, M. Herquet, F. Maltoni, O. Mattelaer and T. Stelzer, JHEP 1106 (2011) 128

[8] V. M. Abazov et al. [D0 Collaboration], Phys. Rev. D 88 (2013) 9, 092001 